\title{
Selective laser ionization of very neutron-rich cadmium isotopes: Decay properties of ${ }^{131} \mathrm{Cd}_{83}$ and ${ }^{132} \mathrm{Cd}_{84}$
}

\author{
M. Hannawald, ${ }^{1}$ K.-L. Kratz, ${ }^{1}{ }^{*}$ B. Pfeiffer, ${ }^{1}$ W. B. Walters,${ }^{2}$ V. N. Fedoseyev, ${ }^{3}$ V. I. Mishin, ${ }^{3}$ W. F. Mueller, ${ }^{4}$ H. Schatz, ${ }^{5}$ \\ J. Van Roosbroeck, ${ }^{4}$ U. Köster, ${ }^{6}$ V. Sebastian, ${ }^{7}$ H. L. Ravn, ${ }^{8}$ and the ISOLDE Collaboration ${ }^{8}$ \\ ${ }^{1}$ Institut für Kernchemie, Universität Mainz, D-55128 Mainz, Germany \\ ${ }^{2}$ Department of Chemistry, University of Maryland, College Park, Maryland 20742 \\ ${ }^{3}$ Institute of Spectroscopy, Russian Academy of Sciences, RU-142092 Troitzk, Russia \\ ${ }^{4}$ Instituut for Kern- en Stralingsfysica, University of Leuven, B-3001 Leuven, Belgium \\ ${ }^{5}$ Gesellschaft für Schwerionenforschung, D-64291 Darmstadt, Germany \\ ${ }^{6}$ Physik Department, TU München, D-85748 Garching, Germany \\ ${ }^{7}$ Institut für Physik, Universität Mainz, D-55128 Mainz, Germany \\ ${ }^{8}$ CERN, CH-1211 Geneva 23, Switzerland \\ (Received 4 May 2000; published 25 September 2000)
}

\begin{abstract}
A chemically selective laser ion source has been applied in a decay study of the very neutron-rich isotopes ${ }^{131} \mathrm{Cd}$ and ${ }^{132} \mathrm{Cd}$ at CERN/ISOLDE. For the $\beta^{-}$decay of the $N=83$ nuclide ${ }^{131} \mathrm{Cd}$ a surprisingly short half-life of $(68 \pm 3) \mathrm{ms}$ and a weak delayed-neutron branch of $P_{n}=(3.5 \pm 1.0) \%$ were observed. For the $N=84$ nuclide ${ }^{132} \mathrm{Cd}$ a half-life of $(97 \pm 10) \mathrm{ms}$ and a $P_{n}$ value of $(60 \pm 15) \%$ were obtained. Schematic features of both decay schemes are developed. We find that our new data are not reproduced by current global models used for $a b$ initio calculations of $\beta$-decay properties without significant changes.

PACS number(s): 27.60. $+\mathrm{j}, 21.10 . \mathrm{Tg}, 21.10 . \mathrm{Pc}, 21.60 .-\mathrm{n}$
\end{abstract}

\section{INTRODUCTION}

Apart from its intrinsic importance to nuclear structure far from stability, the region around the doubly closed-shell nucleus ${ }^{132} \mathrm{Sn}$ is also of astrophysical interest for the formation of the $A \simeq 130$ peak of the solar $r$-process abundance distribution $\left(N_{r, \odot}\right)$. With regard to the single-particle structure, ${ }^{132} \mathrm{Sn}$ itself together with its nearest-neighbor singleparticle (SP, ${ }^{133} \mathrm{Sb}_{82}$ and ${ }_{50}^{133} \mathrm{Sn}_{\mathbf{8 3}}$ ) and single-hole ( $\mathrm{SH}$, ${ }_{49}^{131} \mathrm{In}_{82}$ and $\left.{ }_{50}^{131} \mathrm{Sn}_{\mathbf{8 1}}\right)$ nuclides play an essential role in testing the shell model, and serve as input for any reliable future microscopic nuclear-structure calculations towards the neutron drip line. During the last decade, spectroscopic studies of $\beta^{-}$decays at on-line mass-separator facilities, such as OSIRIS and ISOLDE, have been the most efficient tool to investigate such neutron-rich nuclei. Today, the $\nu$-hole structure of ${ }^{131} \mathrm{Sn}[1]$, the $\pi$-particle structure of ${ }^{133} \mathrm{Sb}[2]$, and the lowest $\nu$-particle states in ${ }^{133} \mathrm{Sn}$ [3] are fairly well known. The only missing information is the $\pi$-hole structure in ${ }^{131} \mathrm{In}$, where so far only the energy of the lowest-lying $\pi p_{1 / 2} \mathrm{SH}$ isomeric state is known within $\pm 25 \mathrm{keV}[1,4]$. The full set of $l=1$ and $l=3 \pi \mathrm{SH}$ levels can in principle be studied through $\beta$ decay of the $N=83, J^{\pi}=7 / 2^{-}$nucleus ${ }^{131} \mathrm{Cd}$ or via $\beta$-delayed neutron $(\beta d n)$ decay of the even more exotic even-even isotope ${ }^{132} \mathrm{Cd}$.

Also in the context of studying details of the $r$-process nucleosynthesis in the $A \simeq 130 N_{r, \odot}$ region, experimental information on nuclear quantities such as masses, $\beta$-decay properties, and general shell-structure features in the vicinity of the classical, neutron-magic $(N=82)$ ' waiting-point' nu-

*Electronic address: klkratz@mail.kernchemie.uni-mainz.de clei below ${ }^{132} \mathrm{Sn}$ is of special interest $[5,6]$. In the $\mathrm{Cd}$ isotopic chain, both the rather long half-life $\left(T_{1 / 2} \simeq 165 \mathrm{~ms}[7,8]\right)$ of $N=82{ }^{130} \mathrm{Cd}$ and the low neutron separation energy $\left(S_{n}\right.$ $\simeq 2 \mathrm{MeV}$ [9-11]) in $N=83{ }^{131} \mathrm{Cd}$ will considerably slow down the $r$-matter flow through the $A \simeq 130$ mass region for neutron-density and temperature conditions of $n_{n}$ $\leqslant 10^{23} \mathrm{~cm}^{-3}$ and $T \simeq 10^{9} \mathrm{~K}$. Under these stellar conditions, the $r$ process will branch off from the $N=82$ shell at $Z$ $=49$, i.e., at ${ }^{131,133} \mathrm{In}$. For higher neutron densities, the breakthrough will already occur at $Z=48$, i.e., in the $\mathrm{Cd}$ chain, and $N=84{ }^{132} \mathrm{Cd}$ will become an $r$-process waiting point. Consequently, the $N \geqslant 82 \mathrm{Cd}$ isotopes will to a large extent determine the $N_{r, \odot}$ peak shape and the total duration of a "canonical", $r$ process (see, e.g., [12]).

\section{EXPERIMENT}

Previous studies of the $\beta$-decay properties of $N=50$ and $N=82$ waiting-point nuclei at the old SC-ISOLDE using a chemically nonselective plasma ion source were-apart from the very low production yields - strongly complicated by isobar and molecular-ion contaminations [7,13]. Because of these experimental problems, recent progress to study $r$-process isotopes has benefited from the selectivity in their production and detection, in particular by applying $Z$-selective resonance-ionization laser ion-source (RILIS) systems at the new Proton Synchrotron Booster (PSB) ISOLDE (see, e.g., [14-17]). In the case of cadmium, experiments to find a well-suited excitation scheme for resonance ionization were initially carried out in Mainz [18] with a laser system similar to the one at ISOLDE. From the analysis of the published atomic levels of Cd (see Ref. [7] in [18]), it became evident that its resonance ionization using the ra- 


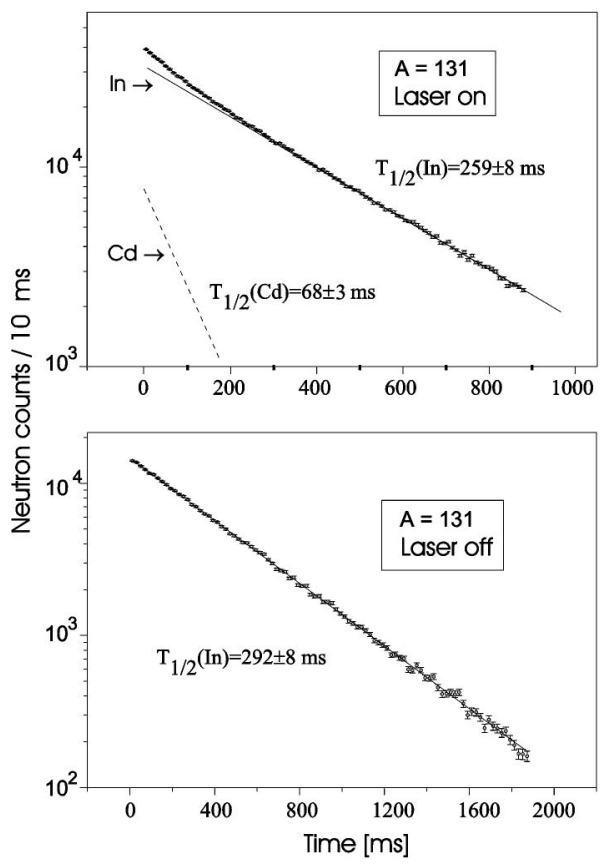

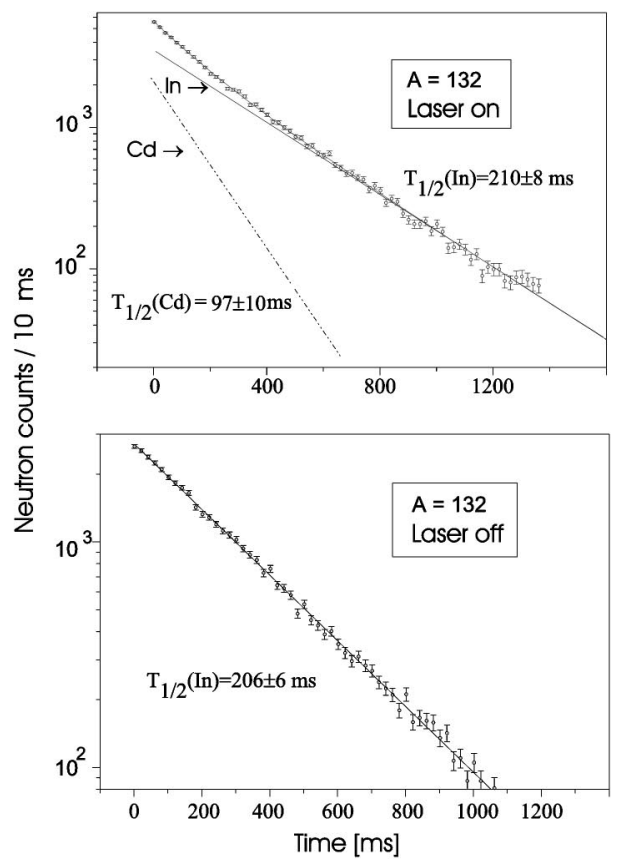

FIG. 1. Beta-delayed neutron decay curves for $A=131$ (left part) and $A=132$ (right part), when operating the ion source in laser-ionization ("laser on," upper parts) or surface-ionization ("laser off,"' lower parts) mode. The "laser off" multiscaling curves represent half-life mixtures of the known In isobars [19]; the "laser on" spectra show a shortlived $\beta d n$ component on top of the respective In activity, which can be attributed to laser-ionized Cd. diation of dye lasers pumped by copper-vapor lasers was possible in three steps. The ionization scheme included two resonant transitions and a third nonresonant excitation into the ionization continuum. As the ionization potential of $\mathrm{Cd}$ is rather high $(8.99 \mathrm{eV})$, laser light in the deep UV is required for the first excitation step (i.e., a wave length of $\lambda_{1}$ $=228.8 \mathrm{~nm}$ ). With the laser system used, this can only be achieved by frequency tripling. The standard method for the generation of such a third harmonic is the combination of two subsequent nonlinear processes of second order: i.e., frequency doubling of the fundamental wavelength $\left(\lambda_{F}\right.$ $=686.4 \mathrm{~nm}$ ) in a nonlinear crystal, followed by sumfrequency mixing of the fundamental and its second harmonic in a second nonlinear crystal. For both steps, BBO (barium- $\beta$-borate) crystals for type-I phase matching (ooe) were used. For further details on the resonance ionization of Cd, we refer to Ref. [18]. Additional information about the RILIS system installed at ISOLDE is given, for example, in Refs. [14,15].

With the above RILIS, a first nuclear-physics experiment to study neutron-rich $\mathrm{Cd}$ nuclides has been carried out at the General Purpose Separator (GPS) of CERN/ISOLDE. The $\mathrm{Cd}$ isotopes were produced by $1-\mathrm{GeV}$ proton-induced fission of a standard uranium-carbide/graphite target $(20 \mathrm{~cm}$ long, thickness $52 \mathrm{~g} / \mathrm{cm}^{2}$ of ${ }^{238} \mathrm{U}$, and about $10 \mathrm{~g} / \mathrm{cm}^{2}$ of graphite). After laser ionization and mass separation, the beams of $\mathrm{Cd}$ nuclides were transported to a beam line equipped with a moving tape system where $\beta d n$-multiscaling measurements could be performed. Counting took place directly at the point of deposit, and the tape system was moved periodically to minimize the buildup of longer-lived daughter nuclides, and to remove the unavoidable surface-ionized isobaric In and Cs activities. Because the $\mathrm{Cd}$ half-lives being sought were expected in the 100-ms range, data acquisition was initiated by the proton pulses from the CERN-PSB, separated by a multiple of $1.2 \mathrm{~s}$, and continued for $1.0 \mathrm{~s}$ for each cycle.

\section{RESULTS AND DISCUSSION}

Beta-delayed neutron data of high statistical quality were collected by multiscaling measurements using the highefficiency Mainz neutron longcounter, equipped with $50{ }^{3} \mathrm{He}$ proportional counters and a $\Delta E$ plastic $\beta$ detector. As an example, Fig. 1 shows $\beta d n$-decay curves obtained for $A$ $=131$ (left part) and $A=132$ (right part), when operating the ion source in laser-ionization ("'laser on," upper parts) or in surface-ionization ("laser off," lower part) mode. The multiscaling curves measured with "laser off', represent halflife mixtures of the known In isomers [19], weighted with their (unknown) production yields and their (known) $P_{n}$ values [20]. The slightly different half-life fits for the In isotopes presumably have their origin in the different primary yields ("laser off,", direct production in fission) and secondary yields ("laser on," production as $\mathrm{Cd} \beta$-decay daughters) of the respective isomers. In all spectra, a small constant neutron background component of only a few counts/channel has been subtracted.

The "laser-on" multiscaling curves for both $A=131$ and 132 clearly show a "short-lived" $\beta d n$ component on top of the respective In isobar, which can be attributed to laserionized Cd. For the new $N=83$ isotope ${ }^{131} \mathrm{Cd}$ a half-life of $T_{1 / 2}=(68 \pm 3) \mathrm{ms}$ has been obtained, which is unanticipatedly short when compared to current model predictions. Also the $\beta d n$-emission probability of $P_{n} \simeq 3.5 \%$ is surprisingly low when considering the quite large energy window of $\left(Q_{\beta}-S_{n}\right) \simeq 5.5-6.1 \mathrm{MeV}$ [9-11]. For example, calculations for pure Gamow-Teller (GT) decay within the quasiparticle random-phase approximation (QRPA) using folded-Yukawa (FY) SP energies and wave functions and a Lipkin-Nogami (LN) microscopic pairing model $[21,22]$ yield $T_{1 / 2}(\mathrm{GT})$ $=943 \mathrm{~ms}$ and $P_{n}(\mathrm{GT}) \simeq 99 \%$ when using $Q_{\beta}=11.6 \mathrm{MeV}$ and $S_{n}=6.2 \mathrm{MeV}$ from the finite-range droplet mass model (FRDM) [9]. The question now arises whether our experi- 
mental results for these two gross $\beta$-decay quantities can be understood in terms of already known $\beta$-strength $\left[S_{\beta}\left(E_{i}\right)\right]$ and nuclear-structure properties in the region of the doublymagic nucleus ${ }^{132} \mathrm{Sn}$.

Generally speaking, the $\beta$-decay half-life $\left(T_{1 / 2}\right)$ is connected to the $\beta$-strength function by the expression

$$
1 / T_{1 / 2}=\sum_{0}^{Q_{\beta}} S_{\beta}\left(E_{i}\right) f\left(Z, Q_{\beta}-E_{i}\right)
$$

where $f\left(Z, Q_{\beta}-E_{i}\right)$ is the Fermi function. With this definition, $T_{1 / 2}$ may yield information on the average $\beta$ feeding of a nucleus. However, the low-energy part of its excitation spectrum is strongly weighted because of the energy factor of $\beta$ decay, $f \sim\left(Q_{\beta}-E_{i}\right)^{5}$. Hence, $T_{1 / 2}$ is dominated by the lowest-energy resonances in $S_{\beta}$, i.e., by the (near-)groundstate (g.s.) allowed (GT) or first-forbidden (ff) transitions.

The $\beta$-delayed neutron-emission probability $\left(P_{n}\right)$ is schematically given by

$$
P_{n}=\frac{\sum_{B_{n}}^{Q_{\beta}} S_{\beta}\left(E_{i}\right) f\left(Z, Q_{\beta}-E_{i}\right)}{\sum_{0}^{Q_{\beta}} S_{\beta}\left(E_{i}\right) f\left(Z, Q_{\beta}-E_{i}\right)},
$$

thus defining $P_{n}$ as the ratio of the integral $\beta$ strength to neutron-unbound states and the total $\beta$ strength. Again, because of the $\left(Q_{\beta}-E_{i}\right)^{5}$ dependence of the Fermi function, the physical significance of the above quantity is limited. It mainly reflects the $\beta$ feeding to the energy region just beyond the neutron separation energy $\left(S_{n}\right)$.

Taken together, however, the two gross $\beta$-decay properties $T_{1 / 2}$ and $P_{n}$ may well provide some first information about the nuclear structure determining $\beta$ decay. There are a number of examples in the literature, where the successful application of this concept has been demonstrated; for example, the development of nuclear structure in the $50 \leqslant N$ $\leqslant 60$ region of the $\mathrm{Sr}$ isotopes [23] or the prediction of collectivity of neutron-magic $(N=28){ }^{44} \mathrm{~S}$ situated two proton pairs below doubly magic ${ }^{48} \mathrm{Ca}[24,25]$.

With the energy sensitivity discussed above, in the present case of ${ }^{131} \mathrm{Cd}_{83}$, our measured integral $\beta$-decay properties lead to two general conclusions. First, there must be significant $\beta$ strength to levels at low energy in the daughter ${ }^{131} \mathrm{In}$, which drives the total half-life. Second, the theoretical high $P_{n}$ value arises because the calculated positions of the three-quasiparticle (3QP) states strongly populated in GT decay lie above $S_{n}$. As a high $P_{n}$ value is not observed, it can be concluded qualitatively that a considerable amount of the GT strength must be located below the neutronseparation energy.

A somewhat similar picture arises for the new $N=84$ isotope ${ }^{132} \mathrm{Cd}$, where the experimental half-life of $T_{1 / 2}=(97$ $\pm 10) \mathrm{ms}$ is again considerably shorter than the predicted $T_{1 / 2}(\mathrm{GT})=633 \mathrm{~ms}$ [22], when using $Q_{\beta}=10.65 \mathrm{MeV}$ from FRDM. Because of the low $S_{n}=2.7 \mathrm{MeV}$ in the ${ }^{132}$ In daughter, the calculated $\beta d n$ branch is $P_{n}(\mathrm{GT}) \simeq 100 \%$ in this case, in qualitative agreement with the rather large experimental value of about $60 \%$. It is interesting to note in this context that the sudden increase in the $\beta d n$ branch between the $N=83$ and $N=84$ isotones of ${ }_{48} \mathrm{Cd}$ is similar to what has been observed earlier for the ${ }_{49}$ In isotones [19]. Such an increase in the $P_{n}$ value is not unexpected as the $\beta$ decay of these $N=83$ nuclides proceeds to $N=82$ closed-shell daughter nuclei that will have a high $S_{n}$ value; whereas in the decay of the $N=84$ isotopes to their $N=83$ daughter nuclides the $S_{n}$ for the single neutron beyond $N=82$ will be low.

In trying to understand the underlying structure of the half-lives and $\beta d n$ branches of these $\mathrm{Cd}$ isotopes on the basis of the nuclear properties of neighboring $N \simeq 82 \mathrm{nu}$ clides, three quantities are of interest: (i) the $Q$ value for $\beta$ decay, (ii) realistic $\log (f t)$ values for the main GT and ff transitions, and (iii) the positions of those states in the daughter nuclei that will be strongly populated. In the following, we will first discuss the importance of these quantities in some detail in the example of ${ }^{131} \mathrm{Cd}_{83}$ decay.

The $Q_{\beta}$ value of ${ }^{131} \mathrm{Cd}$ is predicted by global mass models to lie between $11.5 \mathrm{MeV}$ and $12.6 \mathrm{MeV}$, with the general tendency in this mass region that those models which exhibit a strong $N=82$ shell closure (such as the FRDM [9] or the original extended Thomas-Fermi Strutinski-integral model ETFSI-1 [10]) give the "lower" values. Recent experimental trends in the ${ }^{132} \mathrm{Sn}$ area [26], however, seem to favor the $Q_{\beta}$ predictions from mass models with shell quenching such as HFB/SkP [27], ETFSI-Q [11], or the infinite nuclear matter (INM) model [28]. As can be seen from Fig. 2, between the sequence of ${ }_{50} \mathrm{Sn}$ and ${ }_{48} \mathrm{Cd}$ isotopes there is a significant change in the trend of the experimental and theoretical mass differences, here normalized to the FRDM predictions $\left(M_{i}\right.$ $\left.-M_{F R D M}\right)$. For the $\mathrm{Sn}$ isotopic chain, except maybe ETFSI-1 below $N \simeq 69$ and above $N \simeq 83$, all mass models reproduce the experimental values fairly well within \pm 0.5 $\mathrm{MeV}$. For the $\mathrm{Cd}$ chain, however, clearly the best agreement with the measured masses is obtained with the "quenched" mass models, in particular ETFSI-Q. Therefore, any discussion of theoretical $\beta$-decay properties based on "unquenched" mass models in this region should be regarded with caution (in this context, see, e.g., [29]).

\section{A. Decay of $N=83^{131} \mathrm{Cd}$}

For the case of ${ }^{131} \mathrm{Cd}$, the choice of $Q_{\beta}=12.56 \mathrm{MeV}$ from the ETFSI-Q formula (i.e., about $1 \mathrm{MeV}$ higher than the FRDM prediction) results already in a reduction of the theoretical $T_{1 / 2}$ for GT decay by about a factor of 3 , however still being too long by a factor of 5 . For the GT and ff decays of the $g_{7 / 2}, h_{11 / 2}, s_{1 / 2}$, and $d_{3 / 2}$ neutrons that fill the $N$ $=82$ closed shell, the $\log (f t)$ values may be compared with those from the decay of the isomers of ${ }^{131}$ In to levels in ${ }^{131} \mathrm{Sn}$ as reported by Fogelberg and Blomqvist [1] and listed in [19]. The principal GT-decay branch in the ${ }^{132} \mathrm{Sn}$ region is the $\nu g_{7 / 2} \rightarrow \pi g_{9 / 2}$ transition with a $\log (f t) \simeq 4$.4. This particular decay branch is equivalent to the decay of ${ }^{132} \mathrm{In}$ to a level at $7211 \mathrm{keV}$ in ${ }^{132} \mathrm{Sn}$ whose $\log (f t)$ value is indeed 4.4 [30]. Since this is the first study of a nuclide in which the $f_{7 / 2}$ 

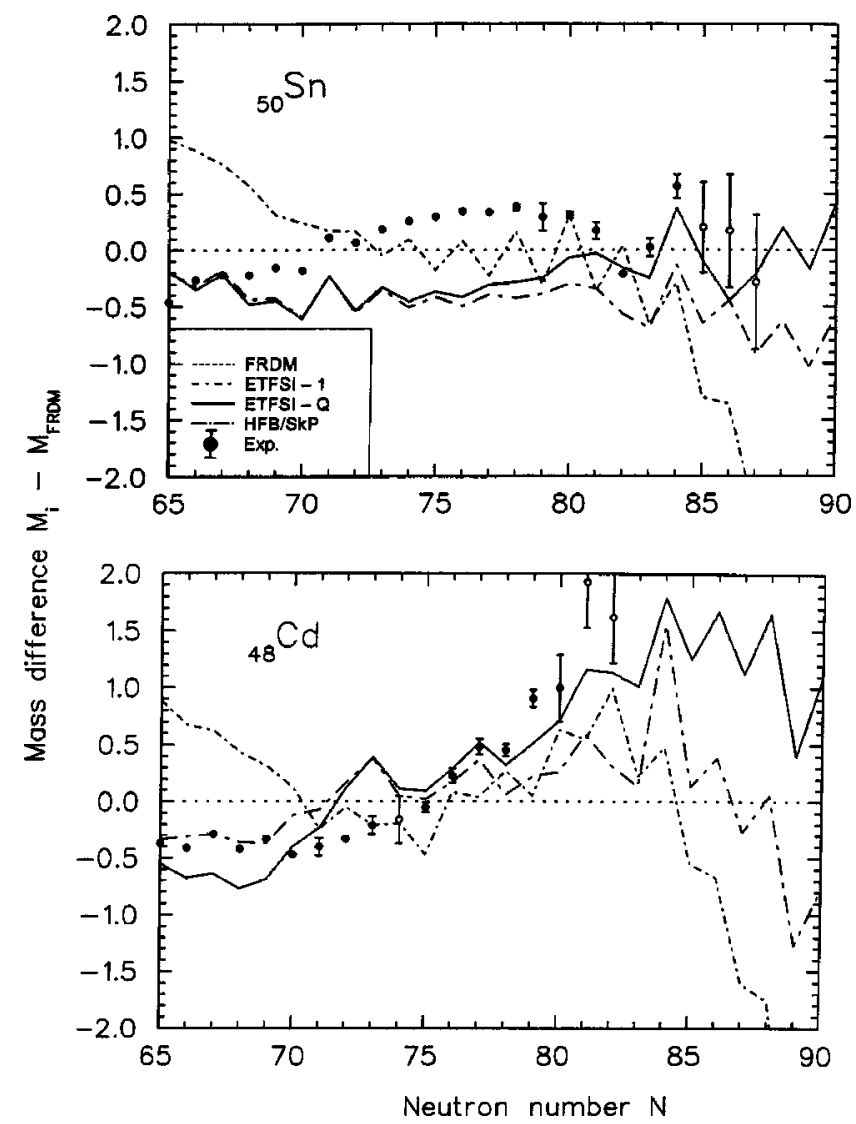

FIG. 2. Experimental [26] and theoretical [10,11,27] mass differences for ${ }_{50} \mathrm{Sn}$ and ${ }_{48} \mathrm{Cd}$ isotopes, normalized to FRDM values [9]. It should be noted that the "data" points for the three (Sn) or two $(\mathrm{Cd})$ heaviest isotopes in both mass chains are values estimated by Audi et al. [26] from local systematic trends in neighboring nuclides. For discussion, see text.

neutron can undergo GT decay to populate the filled $f_{5 / 2}$ proton orbital $\left(\nu f_{7 / 2} \rightarrow \pi f_{5 / 2}\right)$, there are no data available that would permit a direct estimate of this $\log (f t)$ value. Our QRPA calculations show this GT decay strongly, but not completely blocked by the almost complete occupancy of the $f_{5 / 2}$ orbital. We therefore use an estimated $\log (f t)>6.5$ for this transition. The main $\mathrm{ff}$ branches, on the other hand, are the $\nu f_{7 / 2}$ or $\nu h_{11 / 2} \rightarrow \pi g_{9 / 2}$ decays and the $\nu d_{3 / 1}$ or $\nu s_{1 / 2}$ $\rightarrow \pi p_{1 / 2}$ transitions. In the case of ${ }^{131} \mathrm{Cd}$, the above $\nu f_{7 / 2}$ $\rightarrow \pi g_{9 / 2}$ ff-decay branch represents the g.s. to g.s. transition. We can estimate its $\log (f t)$ value from the decay of ${ }^{133} \mathrm{Sn}$ where the single $f_{7 / 2}$ neutron decays to the open $\pi g_{7 / 2}$ and $d_{5 / 2}$ orbitals with $\log (f t)$ of 5.4 and 6.0 , respectively, as reported by Sanchez-Vega et al. [2]. Other forbidden branches in this mass region also show $\log (f t)$ values in this range [19]. Therefore, in analogy to those known cases, we use $\log (f t)=5.4$ for the $\mathrm{ff}$ decay of ${ }^{131} \mathrm{Cd}$ to the g.s. of ${ }^{131} \mathrm{In}$. Its estimated partial half-life of $T_{1 / 2}(\mathrm{ff}) \simeq 150 \mathrm{~ms}$ (which translates into a $\beta$-branching of $\simeq 50 \%$ ) thus turns out to be more important for the total $\beta$-decay half-life than the main GT branches to the $\left[\nu f_{7 / 2}^{+1}\left(\nu g_{7 / 2}^{-1} \pi g_{9 / 2}^{-1}\right)\right]$ 3QP states in ${ }^{131} \mathrm{In}$. Hence, it is quite obvious that ff transitions cannot be neglected in the ${ }^{132} \mathrm{Sn}$ region. Therefore, any straightforward comparisons of experimental $\beta$-decay quantities (like $T_{1 / 2}$ and $P_{n}$ ) with model predictions limited to the GT part of $\beta$ decay (as, for example, explicitly cited in [22]) should be regarded with some caution. In particular, such comparisons should not be overrated to "validate" the reliability and predictive power of such theories (see, e.g., $[31,32]$ ).

To describe the $\beta$ decay of $N=83{ }^{131} \mathrm{Cd}$ correctly, we now must add the main decay branches described above. With this, our model predicts a total $\beta$-decay half-life of $T_{1 / 2}(\mathrm{GT}+\mathrm{ff}) \simeq 195 \mathrm{~ms}$. However, the theoretical $T_{1 / 2}$ is still too long by a factor of 3 , and - even more important-the predicted $P_{n}$ value is with roughly $60 \%$ still much too high compared to $P_{n}($ expt $) \simeq 3.5 \%$. As it is very unlikely that the $\log (f t)$ values in ${ }^{131} \mathrm{Cd}$ decay are considerably smaller than in nearby known decays [19] and predicted by our QRPA, and that another sizable increase in $Q_{\beta}$ beyond the predictions of $[11,27]$ occurs, the only solution is a significantly lower energy for the states strongly populated in the ${ }^{131} \mathrm{Sn}$ daughter. In particular, the lowest $3 \mathrm{QP}$ state fed by the $\nu g_{7 / 2} \rightarrow \pi g_{9 / 2}$ GT transition, which is calculated in the QRPA to lie at $6.96 \mathrm{MeV}$, must have been shifted down in energy below $S_{n} \simeq 6.3 \mathrm{MeV}[11,26]$ in order to explain the small experimental $P_{n}$ value.

Because we cannot account for the required energy shifts within our standard QRPA(FY/LN) model [21], we have replaced the $\nu \mathrm{SP}$ energies from the FY potential by those from other potentials, i.e., a Woods-Saxon model, the classical Nilsson model with different parametrizations [33-35], the relativistic mean-field (RMF) theory with the nonlinear interactions NL1 and NLSH [36,37], and the recent HF/SKX Skyrme method of [38]. The neutron and proton SH states from these theories which are most relevant for our calculations are listed in Table I, together with the experimentally known levels in ${ }^{131} \mathrm{Sn}_{81}$ and ${ }_{49}^{131} \mathrm{In}$. Furthermore, as an alternative to the LN pairing model commonly used in our QRPA calculations, we also applied a BCS approximation as described in [21]. But none of these combinations yielded the required changes relative to the "normal" shell-structure description of ${ }^{132} \mathrm{Sn}$. This is (to a large extent) due to the missing proton-neutron $(p n)$ residual interaction in the QRPA model of [21]. However, even when placing the $\nu g_{7 / 2}$ orbital at the "correct" (experimentally known) position or by choosing the new Nilsson parameters suggested by Zhang et al. [35] - thus simulating the effect of the $\left[\nu g_{7 / 2} \pi g_{9 / 2}\right]$ residual interaction (see, e.g., [39]) — the above 3QP state could not be shifted below the $S_{n}$ value in ${ }^{131} \mathrm{Sn}$. Hence, there must be an additional effect, presumably specific to extremely neutron-rich nuclei. As, e.g., suggested in [40] for isotopes near the neutron drip line, we finally have used a modified Nilsson potential with a reduction of the $l^{2}$ term in the "standard" parametrizations of $[33,34]$ by $25 \%$. Such a reduced $l^{2}$ term in the Nilsson Hamiltonian also leads to a lowered paring energy for the neutrons in this mass region, in our case resulting in $\Delta_{n} \simeq 0.57 \mathrm{MeV}$ instead of the "standard" BCS value of $0.73 \mathrm{MeV}$ in this mass region. This effect would, for example, be consistent with the low energies observed for the $2^{+}(726 \mathrm{keV}), 4^{+}(1073 \mathrm{keV})$, and $6^{+}$ $(1274 \mathrm{keV})$ levels in $N=84{ }^{134} \mathrm{Sn}[41]$. 
TABLE I. Relative level energies of neutron single-hole ( $\nu \mathrm{SH})$ states of ${ }^{131} \mathrm{Sn}_{81}$ and proton single-hole $(\pi \mathrm{SH})$ states of ${ }_{49}^{131} \mathrm{In}$ from different theories, together with experimentally known levels.

\begin{tabular}{lccccccccc}
\hline \hline & Orbital & Expt. & FY & WS & Nilss. [33] & Nilss. [34] & RMF/NL1 & RMF/NLSH & HF/SKX \\
\hline$\nu \mathrm{SH}$ & $d_{3 / 2}$ & 0 & 0 & 0 & 0 & 0 & 0 & 0 & 0 \\
$\left({ }^{131} \mathrm{Sn}\right)$ & $h_{11 / 2}$ & -0.242 & +0.309 & +0.655 & +0.332 & -0.039 & +2.019 & +0.446 & +0.862 \\
& $s_{1 / 2}$ & -0.332 & -0.243 & -0.237 & -1.062 & -0.359 & +0.308 & +0.461 & -0.005 \\
& $d_{5 / 2}$ & -1.655 & -1.942 & -1.817 & -3.186 & -2.768 & -1.623 & -1.759 & -1.856 \\
& $g_{7 / 2}$ & -2.434 & -1.942 & -1.549 & -0.681 & -1.908 & -2.309 & -4.224 & -1.514 \\
$\pi \mathrm{SH}$ & $g_{9 / 2}$ & 0 & 0 & 0 & 0 & 0 & 0 & 0 & 0 \\
$\left({ }^{131} \mathrm{In}\right)$ & $p_{1 / 2}$ & $-0.365^{\mathrm{a}}$ & -0.725 & -0.884 & -0.963 & -0.960 & -1.546 & -0.426 & -1.339 \\
& $p_{3 / 2}$ & $-1.65^{\mathrm{b}}$ & -2.012 & -2.210 & -3.041 & -1.633 & & & -2.513 \\
& $f_{5 / 2}$ & $-2.75^{\mathrm{b}}$ & -3.136 & -3.331 & -1.637 & -3.032 & & & -4.040 \\
\hline \hline
\end{tabular}

${ }^{\mathrm{a}}$ From $\pi p_{3 / 2}$ isomer [19].

${ }^{\mathrm{b}}$ Tentative, from $\gamma$ rays observed in ${ }^{131} \mathrm{Cd}$ decay during this experiment.

And with this procedure, we indeed obtained the required changes: (i) an upward shift of the SP energy of the high$j \nu g_{7 / 2}$ orbital and (ii) a reduction of the $N=82$ shell gap in ${ }^{131} \mathrm{Cd}$ (here defined as $E\left[\nu f_{7 / 2}\right]-E\left[\nu h_{11 / 2}\right]$ ) from about 4.9 $\mathrm{MeV}$ to $3.9 \mathrm{MeV}$. Both effects together result in a downward shift of the excitation energy of the $\left[\nu f_{7 / 2}^{+1}\left(\nu g_{7 / 2}^{-1} \pi g_{9 / 2}^{-1}\right)\right]$ 3QP state below the $S_{n}$ value daughter ${ }^{131} \mathrm{Sn}$. Similarly, all other low-lying 1QP $\left(\pi p_{1 / 2}, \pi p_{3 / 2}\right.$ and $\left.\pi f_{5 / 2}\right)$ and 3QP states are shifted down in energy relative to the initial QRPA(FY/LN) predictions. In consequence, our thus modified QRPA model (with a 25\% reduction of the $l^{2}$ term) now calculates $T_{1 / 2} \simeq 95 \mathrm{~ms}$ and $P_{n} \simeq 3 \%$, in good agreement with the initially surprising experimental values. With a $50 \% l^{2}$ reduction, the GT transition to the lowest $3 \mathrm{QP}$ state would drop further to about $5.4 \mathrm{MeV}$, resulting in a $T_{1 / 2}(\mathrm{GT}+\mathrm{ff})$ $\simeq 70 \mathrm{~ms}$. The decay scheme of $N=83{ }^{131} \mathrm{Cd}$ to ${ }^{131} \mathrm{In}$ proposed according to our above arguments is shown in Fig. 3.

\section{B. Decay of $N=84{ }^{132} \mathrm{Cd}$}

In a similar way as performed for ${ }^{131} \mathrm{Cd}$, we now can discuss the nuclear-structure effects on the $\beta$-decay properties of ${ }^{132} \mathrm{Cd}$. The $Q_{\beta}$ value of this $N=84$ isotope is predicted by recent global mass models to lie between 10.65 $\mathrm{MeV}$ and $12.28 \mathrm{MeV}$, again with the general tendency in the ${ }^{132} \mathrm{Sn}$ region that those models which exhibit a strong $N$ $=82$ shell closure $[9,10]$ give the lower values, in disagreement with the experimental trend shown in Fig. 2. In consequence, the choice of $Q_{\beta}=12.28 \mathrm{MeV}$ from the ETFSI-Q formula (i.e., about $1.6 \mathrm{MeV}$ higher than the FRDM prediction) results already in a reduction of the theoretical $T_{1 / 2}$ calculated within our standard QRPA(FY/LN) model [21] for pure GT decay by about a factor of 4.5. Whereas the resulting $T_{1 / 2}(\mathrm{GT}) \simeq 140 \mathrm{~ms}$ comes already close to the experimental value, the predicted $P_{n}(\mathrm{GT}) \simeq 100 \%$ is still too high. The latter value indicates that the GT decay proceeds entirely to levels in the ${ }^{132}$ In daughter that lie above $S_{n}$ $\simeq 2.7 \mathrm{MeV}$. However, already the addition of (low-lying) $\mathrm{ff}$ strength from the gross theory [42], which does not contain any specific nuclear structure, brings both properties down to the experimental values, i.e., $T_{1 / 2}(\mathrm{GT}+\mathrm{ff}) \simeq 85 \mathrm{~ms}$ and $P_{n}(\mathrm{GT}+\mathrm{ff}) \simeq 80 \%$.
Nevertheless, it seems worthwhile to look into the detailed structure involved in ${ }^{132} \mathrm{Cd}$ decay. If we take the experimental $P_{n}$ value to suggest that about $60 \%$ of the decay must proceed to levels above the neutron-separation energy

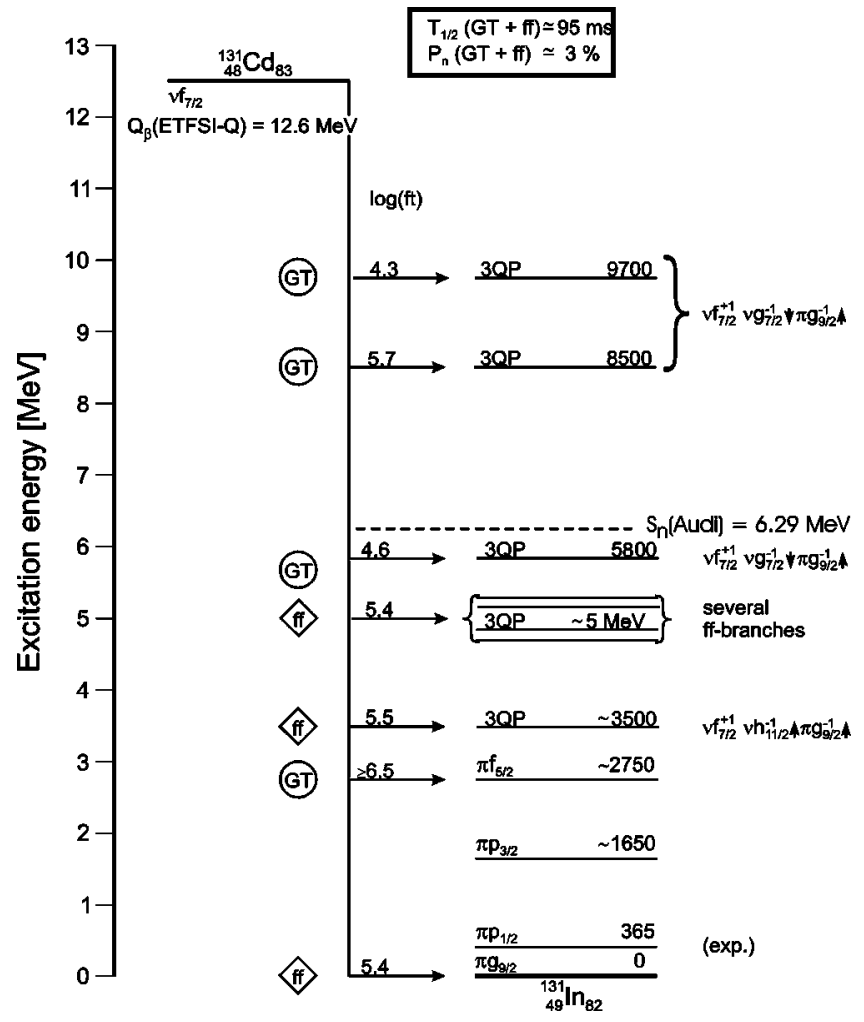

FIG. 3. Proposed decay scheme of $N=83{ }^{131} \mathrm{Cd}$ to ${ }^{131} \mathrm{In}$. The experimentally obtained short $T_{1 / 2} \simeq 68 \mathrm{~ms}$ and the low $P_{n}$ value of $3.5 \%$ cannot be reproduced by current global models restricted to GT decay only. In the case of ${ }^{131} \mathrm{Cd}$, several strong ff branches to low-lying daughter states - in particular the $\nu f_{7 / 2} \rightarrow \pi g_{9 / 2}$ g.s. to g.s. transition-are even more important in determining the above gross $\beta$-decay properties than the allowed transitions. Furthermore, the lowest 3QP state strongly fed in GT decay, which is initially predicted at $6.96 \mathrm{MeV}$, must have been shifted down in energy below $S_{n} \simeq 6.3 \mathrm{MeV}$ in order to explain the very low experimental $P_{n}$ value. For further discussion, see text. 
of $S_{n} \simeq 2.7 \mathrm{MeV}$ and $40 \%$ of the decay must be to levels below $S_{n}$, then we can examine the details of both the GT and ff branches.

The configuration of the $0^{+}$g.s. of ${ }^{132} \mathrm{Cd}$ is $\left[\nu f_{7 / 2}^{+2} \pi g_{9 / 2}^{-2}\right]$. Hence, the main GT-decay branch in this case is expected to be the $\nu f_{7 / 2} \rightarrow \pi f_{5 / 2}$ transition. And indeed, our QRPA(FY/LN) predicts such a $\beta$ branch to a $1^{+} 2 \mathrm{QP}$ state at $5.57 \mathrm{MeV}$ in ${ }^{132} \mathrm{In}$. Calculations with a Woods-Saxon potential predict this level at $5.13 \mathrm{MeV}$, and when using the Nilsson model with a $25 \%$ reduction of the $l^{2}$ term (see discussion for ${ }^{131} \mathrm{Cd}$ above), we can shift the $1^{+}$state further down below $5 \mathrm{MeV}$. In any case, it is quite clear that the $\left[\nu f_{7 / 2} \pi f_{5 / 2}\right] 2 \mathrm{QP}$ state must lie above $S_{n}$. Again, we expect this transition hindered by the high occupancy $(>95 \%)$ of the $\pi f_{5 / 2}$ orbital, and therefore use a $\log (f t) \simeq 5$.4. The second important GT branch is the $\nu g_{7 / 2} \rightarrow \pi g_{9 / 2}$ transition to a $\left[\nu g_{7 / 2} \pi g_{9 / 2}\right] 1^{+}$state, which our QRPA(FY/LN) predicts at 6.76 MeV. In this case, there is some experimental evidence for a lowered position of the $1^{+}$coupling of these spin-orbit partners. The lowest $1^{+}$level in ${ }^{126} \mathrm{In}$ is established at 688 $\mathrm{keV}$, whereas the comparable position of the $\nu g_{7 / 2}$ orbital in ${ }^{127}$ In lies at $1602 \mathrm{keV}[19,43]$. That is, when the $g_{9 / 2}$ proton is removed from ${ }^{127} \mathrm{Sn}$, the position of the resulting $1^{+}$level is about $900 \mathrm{keV}$ below that of the parent $\nu g_{7 / 2}$ orbital [44]. Hence, we assume the respective $\left[\nu g_{7 / 2} \pi g_{9 / 2}\right] 1^{+}$state in ${ }^{132} \mathrm{In}$ around $5.9 \mathrm{MeV}$. The third GT branch which one may expect is the $\nu p_{3 / 2} \rightarrow \pi p_{1 / 2}$ transition to the lowest $1^{+}$level at about $1 \mathrm{MeV}$ in ${ }^{132} \mathrm{In}$. However, so far there are no comparable transitions known in this mass region. In any case, we did not obtain such a transition in our QRPA calculations of ${ }^{132} \mathrm{Cd}$ decay, independent of the SP and pairing models used. The reason is presumably that the $\nu p_{3 / 2}$ level lies too high above the $\nu f_{7 / 2}$ valence orbital in the standard SP models used, resulting in a rather low occupancy of only about $1 \%$. When bringing down the $\nu p_{3 / 2}$ orbital to a value around $850 \mathrm{keV}$ (by using the Nilsson model with a strongly reduced $l^{2}$ term), as experimentally known in the $N=83$ isotone ${ }^{133}$ In [3], we can well increase the occupancy of this level to about $4 \%$, but even then the high occupancy of $\geqslant 90 \%$ of the $\pi p_{1 / 2}$ partner orbital seems to disfavor this GT branch in our model. From these arguments, we estimate that the $\nu p_{3 / 2} \rightarrow \pi p_{1 / 2}$ transition should be rather weak, with a $\log (f t) \geqslant 6.5$.

As far as the ff transitions in ${ }^{132} \mathrm{Cd}$ decay are concerned, from level systematics [19] and nuclear-structure arguments we expect two strong branches, the $\nu f_{7 / 2} \rightarrow \pi g_{9 / 2}$ decay to a low-lying $1^{-}$level around $1 \mathrm{MeV}$ with an estimated $\log (f t)$ $\simeq 5.4$ (as was used for ${ }^{131} \mathrm{Cd}$ decay) and the $\nu h_{11 / 2} \rightarrow \pi g_{9 / 2}$ decay to a high-lying $1^{-}$state at about $5 \mathrm{MeV}$ with a $\log (f t) \simeq 5$.3. While the first of these two ff transitions is of importance for determining both integral $\beta$-decay properties $T_{1 / 2}$ and $P_{n}$, the second one does not influence those values to a considerable extent. For the important $\nu f_{7 / 2} \rightarrow \pi g_{9 / 2} \mathrm{ff}$ decay, which represents the dominating transition to levels below $S_{n}$, we can also use another estimate of its $\beta$ strength. If we assume that this ff branch is responsible for all of the decay to levels below $2.7 \mathrm{MeV}$, corresponding to the experimental value of $\left(100-P_{n}\right) \simeq 40 \%$, it would have a partial

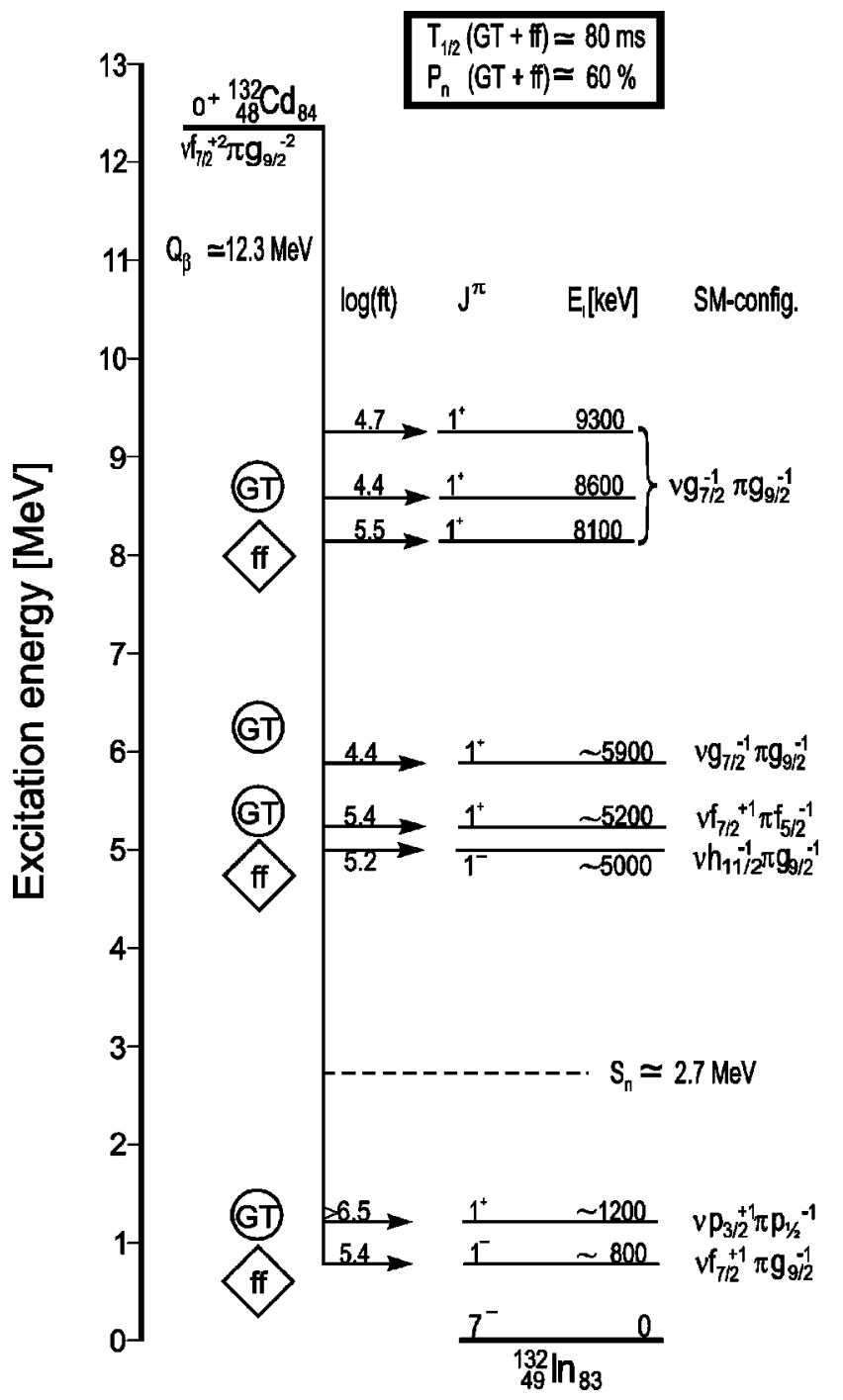

FIG. 4. Proposed decay scheme of $N=84{ }^{132} \mathrm{Cd}$ to ${ }^{132} \mathrm{In}$. As in the case of ${ }^{131} \mathrm{Cd}$, the measured values of the integral $\beta$-decay properties cannot be reproduced by GT decay only. In this case, the short $T_{1 / 2}$ together with the rather high $P_{n}$ value imply that some ff strength (here corresponding to the $\nu f_{7 / 2} \rightarrow \pi g_{9 / 2}$ transition) must lie below $S_{n} \simeq 2.7 \mathrm{MeV}$, but that most of the GT strength goes to neutron-unbound levels of ${ }^{132} \mathrm{In}$. For further discussion, see text.

half-life of roughly $240 \mathrm{~ms}$ and a minimum $\log (f t) \simeq 5.3$, which is in good agreement with the above estimate and the known $\mathrm{ff}$ branches in the ${ }^{132} \mathrm{Sn}$ region.

When adding up the $\beta$-strength contributions of GT and ff decays as discussed above, we obtain a theoretical $T_{1 / 2}$ well below $100 \mathrm{~ms}$ for ${ }^{132} \mathrm{Cd}$ (in the range $75-85 \mathrm{~ms}$ ) and a rather large $P_{n}$ value of about $60 \%$. Both of these values are now well within the uncertainties of our measurements. The decay scheme of ${ }^{132} \mathrm{Cd}$ proposed from the above arguments is shown in Fig. 4.

\section{CONCLUSIONS}

In this paper, we have reported new measurements for the gross $\beta$-decay properties $T_{1 / 2}$ and $P_{n}$ of very neutron-rich $\mathrm{Cd}$ isotopes ${ }^{131,132} \mathrm{Cd}$. These new data provide a basis for esti- 
mates of the distribution of the $\beta$-transition strength to levels in the daughter nuclides ${ }^{131,132} \mathrm{In}$. In turn, these distributions permit some estimates of positions of the important daughter configurations as well as estimates of the decay energies and $\log (f t)$ values needed to drive the observed decays. Several features of the analyses of these numbers stand out. First, the $Q_{\beta}$ values must lie at the upper edges of the previously published theoretical estimates, and indeed provide support for the masses derived from models that include shell quenching, such as the ETFSI-Q or the HFB/SkP approaches. Second, the rather low $\log (f t)$ values of known ff transitions in the ${ }^{132} \mathrm{Sn}$ region seem to be also useful for unknown transitions where the orbital occupancy of the daughter levels is low. Third, the small $P_{n}$ value observed for ${ }^{131} \mathrm{Cd}$ decay requires that the daughter levels whose configuration include the coupling of the $\pi g_{9 / 2}$ g.s. hole and the spin-orbit $\nu g_{7 / 2}$ deep excited hole must lie about $1 \mathrm{MeV}$ below the zerothorder estimate for the usual position of this level.

Finally, these data demonstrate the importance of performing the actual measurements of these half-lives and $\beta$-delayed neutron branchings that are often obtained from theoretical estimates of GT transitions for astrophysical $r$-process calculations. As shown, the published theoretical values for GT decay can easily be an order of magnitude away from the observed values. In this context, we would like to point out that already since the early 1990 s our astrophysics collaboration (see, e.g., [6]) uses an evaluation of $T_{1 / 2}$ and $P_{n}$ values [45], in which the calculated gross properties are based on the FRDM mass and deformation parameters [9] and contain both a microscopic GT strength [22] and a schematic ff strength [42]. For neutron-rich spherical $A \simeq 130$ isotopes, this leads already to shorter $T_{1 / 2}$ and smaller $P_{n}$ values than listed in [22]. The measured values are now available to be incorporated directly into both nuclear-structure and astrophysical calculations, and the insight gained into how these values arise is also of use in making better estimates for other decay properties in this largely unknown mass region southeast of ${ }^{132} \mathrm{Sn}$.

\section{ACKNOWLEDGMENTS}

The authors would like to thank J. Rikovska Stone for many helpful discussions on the possibilities and limitations of present model predictions. We are furthermore indebted to J. Dobaczewski and J.M. Pearson for making available to us their unpublished HFB/SkP and ETFSI-Q mass tables, respectively, and we thank B.A. Brown for sending us his SKX single-particle energies for the ${ }^{132} \mathrm{Sn}$ region. This work was supported by several national foundations, in particular the German BMBF (06MZ864) and DFG (463RUS17/40).
[1] B. Fogelberg and J. Blomqvist, Nucl. Phys. A429, 205 (1984).

[2] M. Sanchez-Vega et al., Phys. Rev. Lett. 80, 5504 (1998); 60, 024303 (1999).

[3] P. Hoff et al., Phys. Rev. Lett. 77, 1020 (1996).

[4] E. Lund et al., Z. Phys. A 294, 233 (1980).

[5] E.M. Burbidge et al., Rev. Mod. Phys. 29, 547 (1957).

[6] K.-L. Kratz et al., Astrophys. J. 402, 216 (1993).

[7] K.-L. Kratz et al., Z. Phys. A 325, 489 (1986).

[8] K.-L. Kratz et al., Hyperfine Interact. (in press).

[9] P. Möller et al., At. Data Nucl. Data Tables 59, 185 (1995).

[10] Y. Aboussir et al., At. Data Nucl. Data Tables 61, 127 (1995).

[11] J.M. Pearson et al., Phys. Lett. B 387, 455 (1996); ETFSI-Q Mass Table (private communication).

[12] K.-L. Kratz et al., Nucl. Phys. A630, 352 (1998).

[13] K.-L. Kratz et al., Z. Phys. A 340, 419 (1991).

[14] V.I. Mishin et al., Nucl. Instrum. Methods Phys. Res. B 73, 550 (1993).

[15] P. Van Duppen, Nucl. Instrum. Methods Phys. Res. B 126, 66 (1997).

[16] K.-L. Kratz, in ENAM 98, edited by Bradley M. Sherrill et al., AIP Conf. Proc. No. 455 (AIP, Woodbury, NY, 1998), p. 827.

[17] M. Hannawald et al., Phys. Rev. Lett. 82, 1391 (1999).

[18] N. Erdmann et al., Appl. Phys. B: Lasers Opt. 66, 431 (1998).

[19] R.B. Firestone et al., Table of Isotopes, 8th ed. (Wiley, New York, 1996).

[20] G. Rudstam et al., At. Data Nucl. Data Tables 53, 1 (1993).

[21] P. Möller and J. Randrup, Nucl. Phys. A514, 1 (1990).

[22] P. Möller et al., At. Data Nucl. Data Tables 66, 13 (1997).

[23] K.-L. Kratz, Nucl. Phys. A417, 447 (1994).

[24] O. Sorlin et al., Phys. Rev. C 47, 2941 (1993).
[25] K.-L. Kratz, A.C. Mueller, and F.-K. Thielemann, Phys. B1. 51, 183 (1995).

[26] G. Audi et al., Nucl. Phys. A624, 1 (1997).

[27] J. Dobaczewski et al., Phys. Scr. T56, 72 (1995); HFB/SkP Mass Table (private communication).

[28] L. Satpathy and R.C. Nayak, At. Data Nucl. Data Tables 39, 241 (1988); J. Phys. G 24, 1527 (1998).

[29] G. Martinez-Pinedo and K. Langanke, Phys. Rev. Lett. 83, 4502 (1999).

[30] B. Fogelberg et al., Phys. Scr. T56, 79 (1995).

[31] F. Ameil et al., Eur. Phys. J. A 1, 275 (1998).

[32] S. Goriely and B. Clerbaux, Astron. Astrophys. 346, 798 (1999).

[33] I. Ragnarsson and R.K. Sheline, Phys. Scr. 29, 385 (1984).

[34] T. Bengtsson and I. Ragnarsson, Nucl. Phys. A436, 14 (1985).

[35] J.-Y. Zhang et al., Phys. Rev. C 58, R2663 (1998).

[36] P. Ring, Prog. Part. Nucl. Phys. 37, 193 (1996).

[37] G.A. Lalazissis et al., Phys. Lett. B 418, 7 (1998); Phys. Rev. C 57, 2294 (1998).

[38] B.A. Brown, Phys. Rev. C 58, 220 (1998).

[39] K.-L. Kratz, in Proceedings of NFFS 6/AMCO 9, edited by R. Neugart and A. Woehr, IOP Conference Series No. 132 (IOP, London, 1993), p. 829.

[40] J. Dobaczewski et al., Phys. Rev. Lett. 72, 981 (1994).

[41] A. Korgul et al., Eur. Phys. J. A 7, 167 (2000).

[42] K. Takahashi et al., At. Data Nucl. Data Tables 12, 101 (1973).

[43] L. Spanier et al., Nucl. Phys. A474, 359 (1987).

[44] W.B. Walters, in Nuclear Fission and Fission-Product Spectroscopy, edited by Gabriele Fioni (AIP, Woodbury, NY, 1998), p. 196.

[45] K.-L. Kratz, B. Pfeiffer, and P. Möller, "Evaluation of $T_{1 / 2}$ and $P_{n}$ values," KCh Report, 1996 (unpublished). 